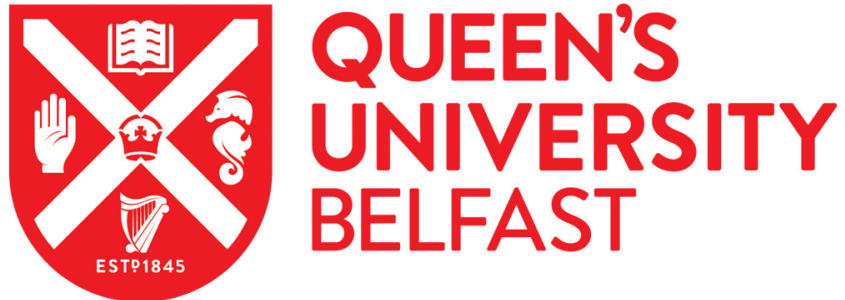

\section{Development and Laboratory Testing of a Multipoint Displacement Monitoring System}

Lydon, D., Taylor, S., Lydon, M., \& Robinson, D. (Accepted/In press). Development and Laboratory Testing of a Multipoint Displacement Monitoring System. Paper presented at Computer Vision Conference, Las Vegas, United States.

Document Version:

Peer reviewed version

Queen's University Belfast - Research Portal:

Link to publication record in Queen's University Belfast Research Portal

Publisher rights

Copyright 2018 The Author.

\section{General rights}

Copyright for the publications made accessible via the Queen's University Belfast Research Portal is retained by the author(s) and / or other copyright owners and it is a condition of accessing these publications that users recognise and abide by the legal requirements associated with these rights.

Take down policy

The Research Portal is Queen's institutional repository that provides access to Queen's research output. Every effort has been made to ensure that content in the Research Portal does not infringe any person's rights, or applicable UK laws. If you discover content in the Research Portal that you believe breaches copyright or violates any law, please contact openaccess@qub.ac.uk. 


\title{
Development and Laboratory Testing of a Multipoint Displacement Monitoring System
}

\begin{abstract}
This paper develops a synchronised multi-camera contactless vision based multiple point displacement measurement system using wireless action cameras. Displacement measurements can provide a valuable insight into the structural condition and service behaviour of bridges under live loading. However conventional displacement gauges or GPS based systems have limitations in terms of access and accuracy. Computer Vision systems have been validated as a means of displacement calculation, however existing systems in use are limited in scope by their inability to reliably track multiple points on a long span bridge structure. The system introduced in this paper provides a low cost durable alternative which is rapidly deployable. Commercial action cameras were paired with an industrially validated solution for synchronisation to provide multiple point displacement readings. The performance of the system was evaluated in a series of controlled laboratory tests. This included the development of robust displacement identification algorithms which were tested and validated against displacement measurements obtained using a fibre optic displacement gauge. This research will significantly advance current vision based Structural health monitoring (SHM) systems which can be cost prohibitive and provides rapid method of obtaining data which accurately relates to measured bridge deflections.
\end{abstract}

KEY WORDS: Computer Vision; Structural Health Monitoring; Bridge Monitoring.

\section{Introduction}

Existing Civil infrastructure is under increasing levels of stress from loading and environmental effects. These effects can be detrimental to the integrity of the bridges and must be monitored to avoid dangerous incidents and ensure public safety. Visual inspections remain to be the most common method of bridge inspection worldwide. This involves a visit to the bridge structure by an inspection team to assess obvious damage to components and is performed according to a set of directives relating to bridge type. This method has many limitations which affect its reliability and is extremely sensitive to human error, particularly since a visual inspection is rarely carried out by a senior engineer. A survey of the reliability of visual inspections has detailed the high level of variability in this assessment method [1].

Structural Health Monitoring (SHM) systems provide a valuable alternative to traditional inspections [2]-[5] and can provide an unbiased and precise means of determining the true state of aging infrastructure. SHM systems allow monitoring of the structural load and response over short periods or for long term, with commonly used sensors including both accelerometers and strain gauges, but there has recently been increased interest in using displacement measurements as a powerful means of assessing bridge condition through performance [6].

Displacement can be measured using traditional sensors such as Linear Variable Differential Transducers (LVDT's). These instruments require contact with the bridge structure to obtain measurements, and an independent and rigid support system, which can be difficult in in many field applications. Accelerometers provide a promising alternative. The drawback with the usage of accelerometers is that they can be vulnerable to numerical error from double integration and initial condition analysis [7]. Laser vibrometers can provide an accurate measurement at a single monitoring location, with the disadvantage of not providing the flexibility of measurement available in vision systems due to being required to be fixed at a single point throughout measurement. Global Position Systems (GPS) can also be used for displacement calculation, but the accuracy of the system is not comparable to that of other systems, with the majority of commercial systems only capable of obtaining a resolution at centimetre resolution and far away from sub millimetre [8]. Traditional sensors also have challenges in evaluating displacement of a structure as a reaction to live loading, or to be accurately synchronized to this loading due to sensor setup and LVDT or slide wire potentiometer internal mechanics.

Computer vision systems for SHM, which primarily consist of single or multiple cameras paired with an image processing algorithm have been in use for structural monitoring since the early 90s [9]. There are several contemporary examples in the literature of the efficacy of computer vision as a tool for SHM. Feng et al [10], developed a low cost contactless system for monitoring of displacement of a bridge structure; where results comparable to LVDT were obtained at distance of approximately $30 \mathrm{~m}$. These readings are useful for displacement calculation; the requirement of having a laptop computer connected to the camera 
used for obtaining video images limits the range of the system. Ferrer et al[11] successfully measured displacement on a concrete surface using consumer grade cameras, but were unable to verify their work under field conditions due to problems with illumination conditions.

There are additional examples of computer vision based displacement calculation described in [12] and [13]. The work discussed above is all in single point displacement calculation, which is limiting on long span bridge structures. Early work in multiple point simultaneous displacement calculation was carried out by Fukuda [14], where multiple PC's were used to control camcorders in a master-slave relationship. This system is based on estimating the time lag between master and slave computers and is always also dependent on the cameras being controlled by PC . This concept is used to determine mode shape of a cable stayed bridge by Chen et al [15], in their research a physical timer is placed in the field of view of each camera at the start of recording, which may not be feasible under field conditions with a long span bridge. Multi- point displacement is also explored in the work of [16], where multiple points in the viewpoint of one camera are used to calculate displacement. This does offer greater flexibility, with the drawback of decreasing the resolution of the system as less detail of the points would be available. The concept of multiple points from a single camera's viewpoint is expanded upon by the work by [17] with results being captured using a high resolution camera- however there are no traditional sensors used as a means of comparison so it is difficult to verify the accuracy of their system when deployed in the field.

This research has shown that multiple camera computer vision systems are viable as a method of displacement calculation. This paper will detail the laboratory trials of a time-synchronised system for multipoint displacement calculation.

\section{System Development}

A feature-based approach was chosen due to being more robust and reliable than DIC approaches[18] and, when paired with a reliable feature extraction technique, with similar precision. The processing framework is composed of three main stages which will be detailed below.

Camera Calibration: Camera Calibration is a method of determining the intrinsic and extrinsic paramenters of the camera used to record the structure motion to remove lens distrortion effects and to provide a scaling factor for the conversion from pixel units to engineering units. The method used in removing lens distortion in this study was the one proposed by Bouguet in [19], where a series of images of a checkerboard or similar pattern is used to obtain the lens distortion of a camera at the desired focal length. There are a variety of approaches used to determine the scaling factor for converting pixels to physical distance. In [20], a pretesting calibration method is demonstrated. This involves setting up the camera in the laboratory in an identical manner to that of the field test to be carried out, i.e. same monitoring distance, focal length, angle etc. The camera is calibrated using the checkerboard pattern, and these variables are used to remove lens distortion and provide a scaling factor for the videos captured in the field trials. The formula to show this is written below in (1)

$$
S F=\frac{d}{D}=\frac{f}{p \times Z}\left(\frac{p i x e l}{m m}\right)
$$

SF is the scaling factor ratio, $d$ is a distance on the image, $D$ is a world distance, $f$ is the focal length of the camera, $p$ is the unit length of the camera sensor ( $\mathrm{mm} / \mathrm{pixel})$ and $Z$ is the distance from the camera to the monitoring location. The scaling factor can also be determined by use of the formula

$$
S F=\frac{D_{\text {Known }}}{I_{\text {Known }}}
$$

where $D_{\text {Known }}$ is the known physical length on the object surface and $I_{\text {Known }}$ is the corresponding pixel length on the image plane.

Feature Extraction: This is the process of extracting/detecting salient features from the images of the object to be tracked.

Examples of these could be corners, rivets or natural decay in a concrete or steel structure. Processing time can be minimised by only searching for features inside a Region of Interest (ROI). The process selected for use in the algorithm was SURF[21], a

robust and computationally inexpensive extension of SIFT[22]. The keypoints provided by SURF are scale and rotation invariant and are detected using a Haar wavelet approximation of the blob(region in an image that differ in properties such as

brightness/colour from surrounding regions) detector based on the Hessian determinant. These approximations are used in combination with integral images(the sum of pixel values in the image) to encode the distribution of pixel intensity values in the

Fig 11. neighborhood of the detected feature. The features detected in the laboratory tests are shown in 


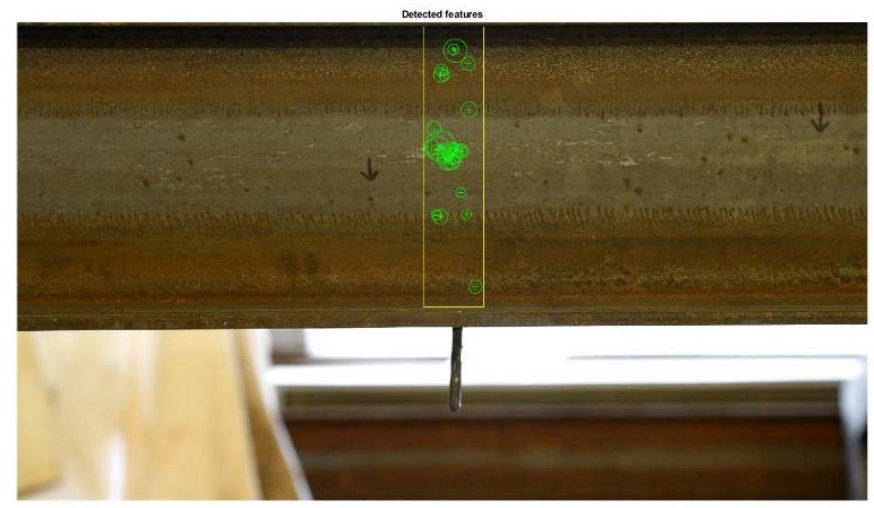

Fig 1 Features Detected in Laboratory Trial

Feature Tracking: Once the points are detected, they must be tracked through subsequent frames to filter outliers and improve the displacement dynamic estimation. Careful application of threshold values must be maintained during this process, as features may become occluded or vary during the progression of a video. Our system makes use of a Kanade- Lucas Tomasi (KLT) [23] tracker to determine movement of the features detected. This method takes the points detected by the feature extractor and uses them as initialisation values. The system removes outliers using the statistically robust M-estimator SAmple Consensus (MSAC) algorithm[24]which is a variant of the RANSAC algorithm. The MSAC algorithm scores inliers according to the fitness to the model and uses this together with a user- specified reprojection error distance to minimize the usage of outliers in the displacement calculation. Any features that do not meet these thresholds are rejected, with the inliers then tracked on the next video frame using the KLT algorithm. The displacement of the object can be measured in pixels by calculating the relative movement between frames of the centroid of a matrix containing the extracted features. The pixel movement is converted to engineering units using the formula in Equation (2). This continues until all frames of the video have been processed.

\subsection{Synchronization Hardware}

The system that was chosen to provide time synchronization for the GoPro systems is known as Syncbac [25]. This GoPro accessory can be attached to the extension port of the GoPro and embeds timecode data into each frame recorded by the GoPro. Analysis of this metadata allows for synchronization of recordings obtained by the system using a solution developed by the authors in $\mathrm{C}++$ in Microsoft Visual Studio. The Syncbac sends live timecode data via Radio Frequency (RF), with a range of 30$60 \mathrm{~m}$. There is also functionality available for units to be initially synchronized with a master unit before handling timecode insertion without any additional information being provided. The range of the system can also be extended to $150-180 \mathrm{~m}$ by use of a pulse: [26], which allows for greater deployment range in addition to wireless control of all units via the Blink Hub app [27]. The Syncbac system also allows for wireless remote control of the cameras via PC/smartphone app, meaning the cameras can be placed in areas not traditionally available for bridge monitoring using Computer Vision.

\section{Experimental Programme}

The aim of the experimental work was to conduct a series of sequential tests to establish the accuracy of the timecode synchronization between the vision sensors, since perfect synchronisation is required for a full characterisation of the deflection pattern

A trial was carried out in the laboratory where two GoPro cameras set to $25 \mathrm{fps}$ were used to monitor the movement of a single target attached to a displacement testing apparatus while it was displaced manually. The cameras were placed $4.3 \mathrm{~m}$ away from the monitoring location and pixel-mm conversion was carried out using dimension correspondences method in Eq. (2). Verification of the measured displacement was provided by an accurately calibrated fibre optic displacement sensor (FOS) with a resolution of $0.03 \mathrm{~mm}$ set to record at $25 \mathrm{hz}$. The wavelength shift data associated with the FOS was recorded and converted to displacement using an approach described previously [28], where a Fabry-Perot filter was used in tandem with a photodiode. The setup for this trial is shown in Fig 2. 


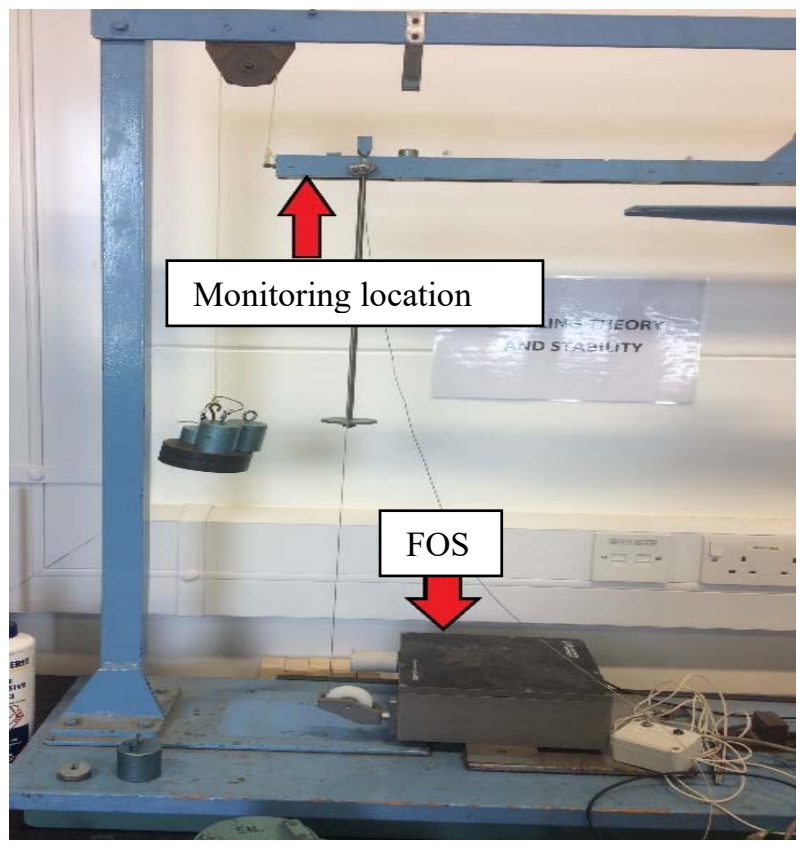

Fig 2 Setup of Displacement Apparatus for multiple cameras at single monitoring location trial

The Root Mean Square Error(RMSE) and Coerrelation coefiicient(CC) in comparison to the validation sensor and between each camera is shown in Table I, with the time-displacement series for all instrumentation plotted in Fig. 3.

TABLE I

ReSUlts: Multiple CAMERAS AT A SINGLE MONITORING LOCATION

\begin{tabular}{|c|c|c|c|c|}
\hline $\begin{array}{c}\text { GoPro } 1 \\
\text { RMSE vs } \\
\text { FOS(mm) }\end{array}$ & $\begin{array}{c}\text { GoPro } 1 \\
\text { CC vs FOS }\end{array}$ & $\begin{array}{c}\text { GoPro } 2 \\
\text { RMSE vs } \\
\text { FOS(mm) }\end{array}$ & $\begin{array}{c}\text { GoPro } 2 \\
\text { CC vs FOS }\end{array}$ & $\begin{array}{c}\text { GoPro } 1 \\
\text { CC vs } \\
\text { GoPro } 2\end{array}$ \\
\hline 1533 & .9914 & .0928 & .9975 & .9869 \\
\hline
\end{tabular}

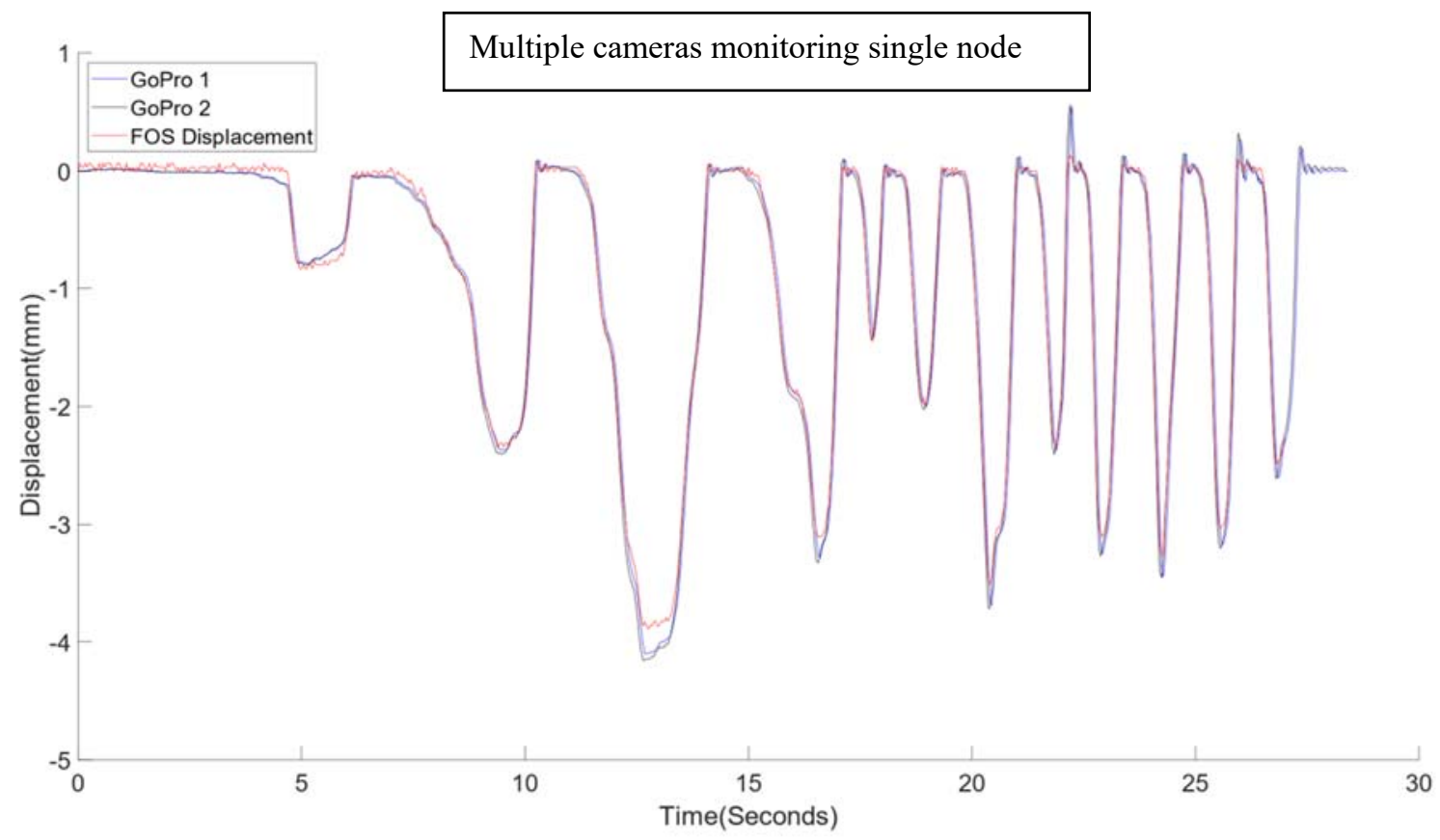

Fig. 3 Results of Multiple Cameras at Single Node Trial 
This trial provided confidence in the capabilities of the system for measuring displacement from multiple synchronised cameras.

\section{Test Series 2 - Accuracy of synchronized vision sensors for displacement measurement at multiple nodes}

The accuracy of the hardware system and associated post processing techniques were further validated through an additional laboratory experimental program. This involved tracking the displacement of a simply supported $178 \mathrm{~mm} \times 102 \mathrm{~mm} \times 19 \mathrm{~mm}$ Universal Beam with a clear span of $5.3 \mathrm{~m}$. A centrally applied static load of $3255 \mathrm{~N}$ was applied to induce displacement along the span of the beam. The beam was split into 9 elements of equal length along the span of them beam to situate the loading and sensing points. The nodes connecting the elements were numbered 1-10 consecutively from left to right with the load applied midway between nodes 5 and 6 .

\section{1) Sensor configuration and data acquisition}

LVDT's were used to validate the camera measurements at the monitoring locations used by the camera along the span. The LVDT sensors were configured to measure static displacements at each of the nodes monitored by the camera. A Datataker DT800 logger was used to acquire the readings at discrete times during the test corresponding to times when the beam was loaded and unloaded. The FOS from Test series 1 was also used at a single node (Node 4) to validate the accuracy of the deflections that were determined from the camera readings. This sensor was used because it provides a high level of accuracy compared to LVDT. The FOS was positioned to record continuous displacement measurements at a rate of $25 \mathrm{~Hz}$. Two GoPros set to record continuously during loading and unloading at a frame rate of $25 \mathrm{fps}$ were used to monitor the beam. One camera was set to monitor node 3 and the second to monitor node 4 . The readings taken at each node were converted from pixels to mm using the scaling factor presented in Equation (2).

The results from the synchronization trial for nodes 3 and 4 are shown in Fig 4 . The results show good agreement between the camera system and the LVDT/FOS sensors, with our vision system outperforming the LVDT sensors in the point where the FOS was available. It is also significant that there is excellent correlation between the vision sensors along the X-axis, confirming the successful synchronization of the system in a laboratory environment.

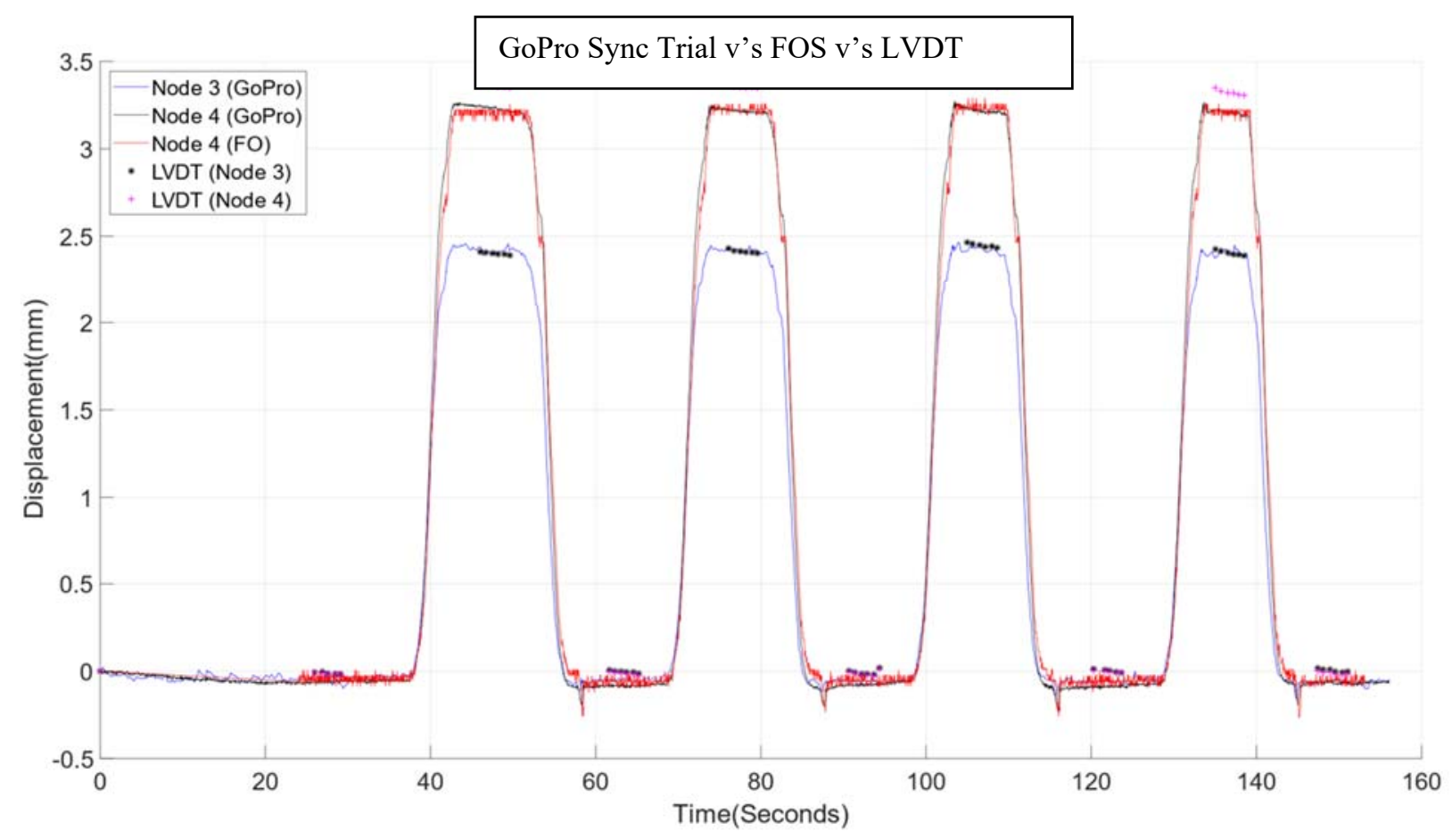

Fig 4 Vision, FOS and LVDT sensor displacement results for test series 2

To allow for accurate comparison between the displacement results calculated from the vision-based sensors and the validation sensors, the RMSE for all 3 validation sensors and the CC for the comparison between FOS and the camera is presented in Table II. 
TABLE II

RESULTS FROM GOPRO VS FOS VS LVDT DISPLACEMENT MONITORING

\begin{tabular}{cccc}
\hline \hline Camera Node & $\begin{array}{c}\text { Validation } \\
\text { Sensor }\end{array}$ & $\begin{array}{c}\text { RMSE (vision sensor v's } \\
\text { validation sensor) }(\mathrm{mm})\end{array}$ & $\begin{array}{c}\text { CC(Vision Sensor vs } \\
\text { Validation Sensor) }\end{array}$ \\
\hline 3 & LVDT & 0.044 & N/A \\
4 & LVDT & 0.107 & .894 \\
4 & FOS & 0.079 & .89 \\
\hline \hline
\end{tabular}

The results confirm successful synchronization of the two cameras, providing confidence in this method for future deployment. It is believed by the authors that the higher RMSE with the LVDT comparison at Node 4 is due to the lower resolution of the LVDT versus the FOS. The low error results from the RMSE comparison validate the use of the GoPro camera in laboratory trials.

\section{Conclusions \& Future work}

A sequential series of tests have been carried out to validate our fully synchronized wireless vision sensor monitoring system. Each of the tests carried out was designed to build upon the results of previous work and facilitated the development of an accurate algorithm for determining displacements from video footage. The results from the vision-based sensor provided excellent correlation with validated measurement methods. The innovative synchronisation of the wireless camera system can, for the first time, facilitate the measurement of a deflection profile along the full span of a bridge without the need for contact with the structure or. This has the potential to provide valuable information for early damage detection which was previously limited to vibration analysis.

A review of the existing literature highlights the need for a precise fully wireless monitoring system which could be rapidly deployed on a bridge of medium to long span. The systems presented overcome previous limitations in terms of cost and power consumption as well as in the size of the infrastructure due to the use of multiple vision sensors. The research so far has developed solutions for multipoint displacement monitoring using cameras. Further work to be carried out to develop a complete system for bridge monitoring due to traffic loading includes:

- Perform field trials of the system with validating equipment to ensure accuracy remains consistent in an uncontrolled environment.

- Develop a machine learning system for traffic identification.

- Correlate vehicle position to applied load in laboratory and field trials.

- Investigate the use of stereo vision to obtain 3D measurement of bridge movement.

- Incorporate applied load position and measured deflection into a system to demonstrate the influence line of a bridge under loading.

\section{References}

[1] B. R. Program, “Tech Notes Reliability of Visual Inspection for Highway Bridges, Volume I : Final Report and , Volume II : Appendices," 2003.

[2] M. Lydon, S. Taylor, D. Robinson, C. Doherty, and P. Callender, “ASSESSMENT OF VARIOUS SENSORS FOR STRUCTURAL HEALTH MONITORING FOR BRIDGE WEIGH - IN - MOTION ( B - WIM ), in The 6 th International Conference on Structural Health Monitoring of Intelligent Infrastructure, 2013, no. December.

[3] D. Inaudi, "Cost-Benefits Analysis in SHM Projects Cost-Benefits Analysis in SHM Projects," Proc. Fifth Int. Conf. Struct. Heal. Monit. Intell. Infrastruct., no. December, pp. 1-9, 2011.

[4] R. Zaurin and F. Necati Catbas, "Structural health monitoring using video stream, influence lines, and statistical analysis," Struct. Heal. Monit. An Int. J., vol. 10, no. 3, pp. 309-332, May 2011.

[5] D. H. Sigurdardottir and B. Glisic, "On-site validation of fiber-optic methods for structural health monitoring: Streicker Bridge,” J. Civ. Struct. Heal. Monit., vol. 5, no. 4, pp. 529-549, 2015.

[6] T. Ojio, C. Carey, E. OBrien, C. Doherty, and S. Taylor, “Contactless Bridge Weigh-in-Motion,” J. Bridg. Eng., p. 4016032, Feb. 2016.

[7] K.-T. Park, S.-H. Kim, H.-S. Park, and K.-W. Lee, "The determination of bridge displacement using measured acceleration," Eng. Struct., vol. 27, no. 3, pp. 371-378, Feb. 2005. 
[8] S. B. Im, S. Hurlebaus, and Y. J. Kang, "Summary Review of GPS Technology for Structural Health Monitoring," J. Struct. Eng., vol. 139, no. 10, pp. 1653-1664, Oct. 2013.

[9] G. A. Stephen, J. M. W. Brownjohn, and C. A. Taylor, "Measurements of static and dynamic displacement from visual monitoring of the Humber Bridge,” Eng. Struct., vol. 15, no. 3, pp. 197-208, Jan. 1993.

[10] M. Q. Feng, Y. Fukuda, D. Feng, and M. Mizuta, "Nontarget Vision Sensor for Remote Measurement of Bridge Dynamic Response," J. Bridg. Eng., vol. 20, no. 12, p. 04015023, Dec. 2015.

[11] B. Ferrer, P. Acevedo, J. Espinosa, and D. Mas, "Targetless image-based method for measuring displacements and strains on concrete surfaces with a consumer camera," Constr. Build. Mater., vol. 75, pp. 213-219, Jan. 2015.

[12] M. H. Shih and W. P. Sung, "Developing dynamic digital image techniques with continuous parameters to detect structural damage," Sci. World J., vol. 2013, p. 453468, 2013.

[13] J.-W. Park, J.-J. Lee, H.-J. Jung, and H. Myung, "Vision-based displacement measurement method for high-rise building structures using partitioning approach," NDT E Int., vol. 43, no. 7, pp. 642-647, Oct. 2010.

[14] Y. Fukuda, M. Q. Feng, and M. Shinozuka, "Cost-effective vision-based system for monitoring dynamic response of civil engineering structures," Struct. Control Heal. Monit., vol. 17, no. 8, pp. 918-936, Dec. 2010.

[15] C. C. Chen, W. H. Wu, H. Z. Tseng, C. H. Chen, and G. Lai, "Application of digital photogrammetry techniques in identifying the mode shape ratios of stay cables with multiple camcorders," Meas. J. Int. Meas. Confed., vol. 75, pp. 134146, Nov. 2015.

[16] S. W. Kim and N. S. Kim, "Multi-point displacement response measurement of civil infrastructures using digital image processing," Procedia Eng., vol. 14, pp. 195-203, Jan. 2011.

[17] D. Feng and M. Q. Feng, "Experimental validation of cost-effective vision-based structural health monitoring," Mech. Syst. Signal Process., vol. 88, pp. 199-211, May 2017.

[18] G. Hong and Y. Zhang, "Combination of feature-based and area-based image registration technique for high resolution remote sensing image," in Geoscience and Remote Sensing Symposium, 2007. IGARSS 2007. IEEE International, 2007, pp. $377-380$.

\begin{tabular}{|c|c|c|c|}
\hline $\begin{array}{c}\text { Bouguet, } \\
\text { //Www.Vision.Cal }\end{array}$ & $\begin{array}{l}\text { "Camera Calibration } \\
\text { s/Bouguetj/Calib_Doc/Index.Html\#Ref, }\end{array}$ & $\begin{array}{r}\text { Toolbox } \\
2015 .\end{array}$ & $\begin{array}{l}\text { for } \\
\text { [Online]. }\end{array}$ \\
\hline
\end{tabular}

[20] T. Khuc and F. N. Catbas, "Computer vision-based displacement and vibration monitoring without using physical target on structures," Struct. Infrastruct. Eng., vol. 13, no. 4, pp. 505-516, Apr. 2017.

[21] H. Bay, T. Tuytelaars, and L. Van Gool, "SURF: Speeded up robust features," in Lecture Notes in Computer Science (including subseries Lecture Notes in Artificial Intelligence and Lecture Notes in Bioinformatics), 2006, vol. 3951 LNCS, pp. 404-417.

[22] D. G. Lowe, "Object recognition from local scale-invariant features," in Proceedings of the Seventh IEEE International Conference on Computer Vision, 1999, pp. 1150-1157 vol.2.

[23] C. Tomasi and T. Kanade, "Detection and Tracking of Point Features," Carnegie Mellon University Technical Report, 1991. [Online]. Available: http://www.lira.dist.unige.it/teaching/SINA/slides-current/tomasi-kanade-techreport-1991.pdf.

[24] P. H. S. Torr and A. Zisserman, "MLESAC: A new robust estimator with application to estimating image geometry," Comput. Vis. Image Underst., vol. 78, no. 1, pp. 138-156, 2000.

[25] Timecode Systems, "SyncBac Pro Home | Timecode Systems." [Online]. Available: https://www.timecodesystems.com/syncbac-pro/. [Accessed: 09-Mar-2018].

[26] Timecode Systems, :"pulse | Timecode Systems.” [Online]. Available: https://www.timecodesystems.com/productshome/pulse/. [Accessed: 09-Mar-2018].

[27] Timecode Systems, "BLINK Hub - Free sync \&amp; control app | Timecode Systems." [Online]. Available: https://www.timecodesystems.com/products-home/blink-hub-timecode-app/. [Accessed: 09-Mar-2018].

[28] M. Lydon et al., "Development of a bridge weigh-in-motion sensor: Performance comparison using fiber optic and electric resistance strain sensor systems," IEEE Sens. J., vol. 14, no. 12, 2014. 